\title{
Quantized State-Feedback Stabilization for Delayed Markovian Jump Linear Systems with Generally Incomplete Transition Rates
}

\author{
Yanbo Li, ${ }^{1}$ Peng Zhang, ${ }^{2}$ Yonggui Kao, ${ }^{3}$ and Hamid Reza Karimi ${ }^{4}$ \\ ${ }^{1}$ School of Mathematical Sciences, Guangxi Teachers Education University, Guangxi 530001, China \\ ${ }^{2}$ School of Materials Science and Engineering, Harbin Institute of Technology, Weihai 264209, China \\ ${ }^{3}$ Department of Mathematics, Harbin Institute of Technology, Weihai 264209, China \\ ${ }^{4}$ Department of Engineering, Faculty of Technology and Science, University of Agder, 4898 Grimstad, Norway
}

Correspondence should be addressed to Peng Zhang; pzhang@yeah.net and Hamid Reza Karimi; hamid.r.karimi@uia.no

Received 19 February 2014; Accepted 13 April 2014; Published 26 May 2014

Academic Editor: Hui Zhang

Copyright (c) 2014 Yanbo Li et al. This is an open access article distributed under the Creative Commons Attribution License, which permits unrestricted use, distribution, and reproduction in any medium, provided the original work is properly cited.

\begin{abstract}
This paper is concerned with the robust quantized state-feedback controller design problem for a class of continuous-time Markovian jump linear uncertain systems with general uncertain transition rates and input quantization. The uncertainties under consideration emerge in both system parameters and mode transition rates. This new uncertain model is more general than the existing ones and can be applicable to more practical situations because each transition rate can be completely unknown or only its estimate value is known. Based on linear matrix inequalities, the quantized state-feedback controller is formulated to ensure the closed-loop system is stable in mean square. Finally, a numerical example is presented to verify the validity of the developed theoretical results.
\end{abstract}

\section{Introduction}

Markovian jump systems have been serving as popular tools for analyzing plants subjected to random abrupt changes, such as random component failures, abrupt environment changes, disturbance, and changes in the interconnections of subsystems; see [1-11] and the references therein. In practice, transition rates are difficult to precisely estimate the transition rates. Therefore, developing the analysis and synthesis method for MJS with uncertain transition rates is of great importance. Bounded uncertain transition rates (BUTRs) are introduced when the precise values of the transition rates are unknown, but their bounds (upper bounds and lower bounds) are known; see, for example, [12-14]. However, in some practical cases, to obtain the bound of every TR is difficult or even impossible. Zhang et al. [15-18] proposed another description for the uncertain TRs, which is partly unknown TRs (PUTRs). In this description, some of the TRs can be unknown. This model also simulates researchers' interests (see, e.g., [19-22]). In this PUTR model, every transition rate is either exactly known or completely unknown, which may be too restrictive in many practical situations. Therefore, Guo and Wang [23] proposed generally uncertain TRs to deal with a more practical situation where the transition rates can be completely unknown or only its bound is known.

On the other hand, in many modern engineering practices, information processing devices, such as analog-todigital and digital-to-analog converters, have been widely used and brought about some advantages, such as lower cost, reduced weight and power, and simple installation and maintenance. However, server deterioration of system performance or even system instability may also be induced. Signal quantization should be fully considered in such cases. Nowadays, the feedback stabilization problem is considered by utilizing dynamic quantizers [24-26] and static quantizers [27-36]. The stabilization problem for single-input discrete Markov jump linear systems via mode dependent quantized state feedback is addressed by Xiao et al. in [37], but the transition rates are assumed to be completely known. Ye et al. $[38,39]$ considered $\mathrm{H}_{2}$ control of Markov jump linear systems 
with unknown transition rates and input quantization. To the best of our knowledge, no result has been presented for control design of delayed continuous-time Markov jump linear uncertain systems with generally unknown transition rates and input signal quantization.

In this paper, robust quantized state-feedback stabilization for delayed Markovian jump linear systems with generally uncertain transition rates is addressed. In Section 2, the considered systems are formulated and the purposes of the paper are stated. In Section 3, the main results are derived via linear matrix inequalities. The structure of the controller consists of two parts. The nonlinear part is provided to eliminate the effect of input quantization. The linear part is obtained by solving LMIs to deal with model uncertainties and unknown transition rates. Section 4 concludes the paper.

Notation. In this paper, $\mathbb{R}^{n}$ and $\mathbb{R}^{n \times m}$ denote the $n$ dimensional Euclidean space and the set of all $n \times m$ real matrices, respectively. $\mathbb{N}^{+}$represents the set of positive integers. The notation $P>0(P \geq 0)$ means that $P$ is a real symmetric and positive-definite (semi-positive-definite) matrix. For notation $(\Omega, \mathscr{F}, P), \Omega$ represents the space, $\mathscr{F}$ is the $\sigma$-algebra of subsets of the sample space, and $P$ is the probability measure on $\mathscr{F} . E\{\cdot\}$ stands for the mathematical expectation. Matrices, if their dimensions are not explicitly stated, are assumed to be compatible for algebraic operations.

\section{Problem Formulation}

Consider the following stochastic system with Markovian jump parameters, defined on a complete probability space $(\Omega, \mathscr{F}, P)$ :

$$
\begin{aligned}
\dot{x}(t)= & A\left(r_{t}\right) x(t)+A_{d}\left(r_{t}\right) x(t-\tau) \\
+ & \left(B\left(r_{t}\right)+\Delta B\left(r_{t}\right)\right) q(u(t)), \quad t \geq 0, \\
& x_{0}=x(0), \quad r_{0}=r(0),
\end{aligned}
$$

where $x(t) \in \mathbb{R}^{n}$ is the system state. The mode jumping process $\left\{r_{t}, t \geq 0\right\}$ is a right-continuous Markov process on the probability space taking values in a finite state space $\mathbb{S}=$ $\{1,2, \ldots, s\}$ with the mode transition probabilities:

$$
\operatorname{Pr}\left\{r_{t+\Delta}=j \mid r_{t}=i\right\}= \begin{cases}\pi_{i j} \Delta+o(\Delta), & \text { if } i \neq j, \\ 1+\pi_{i j} \Delta+o(\Delta), & \text { if } i=j,\end{cases}
$$

where $\Delta>0, \lim _{\Delta \rightarrow 0}(o(\Delta) / \Delta)=0$, and $\pi_{i j} \geq 0(i, j \in \mathbb{S}, j \neq i)$ is the TR from mode $i$ at time $t$ to mode $j$ at time $t+\Delta$, and

$$
\pi_{i i}=-\sum_{j=1, j \neq i}^{s} \pi_{i j}
$$

for each $i \in \mathbb{S}$.

The mode TR matrix $\Pi \triangleq\left(\pi_{i j}\right)$ is considered to be generally uncertain. For instance, the TR matrix for system (1) with $s$ operation modes may be expressed as

$$
\left[\begin{array}{ccccc}
\widehat{\pi}_{11}+\Delta_{11} & ? & \widehat{\pi}_{13}+\Delta_{13} & \cdots & ? \\
? & ? & \hat{\pi}_{23}+\Delta_{23} & \cdots & \widehat{\pi}_{2 s}+\Delta_{2 s} \\
\vdots & \vdots & \vdots & \ddots & \vdots \\
? & \hat{\pi}_{s 2}+\Delta_{s 2} & ? & \cdots & \widehat{\pi}_{s s}+\Delta_{s s}
\end{array}\right],
$$

where $\widehat{\pi}_{i j}$ and $\Delta_{i j} \in\left[-\delta_{i j}, \delta_{i j}\right]\left(\delta_{i j} \geq 0\right)$ represent the estimate value and estimate error of the uncertain TR $\pi_{i j}$, respectively, where $\hat{\pi}_{i j}$ and $\delta_{i j}$ are known. "?" represents the complete unknown TR, which means its estimate value $\widehat{\pi}_{i j}$ and estimate error bound are unknown. For notational clarity, for all $i \in$ $\mathbb{S}$, the set $U^{i}$ denotes $U^{i}=U_{k}^{i} \cup U_{u k}^{i}$ with $U_{k}^{i} \triangleq\{j$ : the estimate value of $\lambda_{i j}$ is known for $\left.j \in \mathbb{S}\right\}, U_{u k}^{i} \triangleq\{j$ : the estimate value of $\lambda_{i j}$ is unknown for $\left.j \in \mathbb{S}\right\}$. Moreover, if $U_{k}^{i} \neq \varnothing$, it is further described as $U_{k}^{i}=\left\{k_{1}^{i}, k_{2}^{i}, \ldots, k_{m}^{i}\right\}$, where $k_{m}^{i} \in \mathbb{N}^{+}$represent the $m$ th bound-known element with the index $k_{m}^{i}$ in the $i$ th row of matrix $\Pi$. According to the properties of the TRs (e.g., $\lambda_{i j} \geq 0(\forall i, j \notin \mathbb{S}, i \neq j)$ and $\lambda_{i i}=$ $-\sum_{j=1, j \neq i}^{N} \lambda_{i j}$ ), we assume that the known estimate values of the TRs are well defined. That is, the following assumptions hold.

Assumption 1. If $U_{k}^{i}=\mathbb{S}$, then $\hat{\lambda}_{i j}-\delta_{i j} \geq 0(\forall j \in$ $\mathbb{S}, j \neq i), \hat{\lambda}_{i i}=-\sum_{j=1, j \neq i}^{N} \hat{\lambda}_{i j} \leq 0$, and $\delta_{i i}=\sum_{j=1, j \neq i}^{N} \delta_{i j}>0$.

Assumption 2. If $U_{k}^{i} \neq \mathbb{S}$, and $i \in U_{k}^{i}$, then $\hat{\lambda}_{i j}-\delta_{i j} \geq 0(\forall j \in$ $\left.U_{k}^{i}, j \neq i\right), \widehat{\lambda}_{i i}+\delta_{i i} \leq 0$ and $\sum_{j \in U_{k}^{i}} \widehat{\lambda}_{i j} \leq 0$.

Assumption 3. If $U_{k}^{i} \neq \mathbb{S}$ and $i \notin U_{k}^{i}$, then $\widehat{\lambda}_{i j}-\delta_{i j} \geq 0(\forall j \in$ $\left.U_{k}^{i}\right)$.

Remark 4. The above assumption is reasonable, since it is the direct result from the properties of the TRs

$$
\text { (e.g., } \left.\pi_{i j} \geq 0(\forall i, j \notin \mathbb{S}, i \neq j), \pi_{i i}=-\sum_{j=1, j \neq i}^{s} \pi_{i j}\right) \text {. }
$$

The above description about uncertain TRs is more general than either the BUTR or PUTR models. To show this, we rewrite the two uncertain models as follows: BUTR model (see [12-14]):

$$
\left[\begin{array}{cccc}
\hat{\pi}_{11}+\Delta_{11} & \hat{\pi}_{12}+\Delta_{12} & \cdots & \hat{\pi}_{1 s}+\Delta_{1 s} \\
\hat{\pi}_{21}+\Delta_{21} & \hat{\pi}_{22}+\Delta_{22} & \cdots & \widehat{\pi}_{2 s}+\Delta_{2 s} \\
\vdots & \vdots & \ddots & \vdots \\
\hat{\pi}_{s 1}+\Delta_{s 1} & \hat{\pi}_{s 2}+\Delta_{s 2} & \cdots & \hat{\pi}_{s s}+\Delta_{s s}
\end{array}\right]
$$

with $\widehat{\pi}_{i j}-\delta_{i j} \geq 0(\forall j \in \mathbb{S}, j \neq i), \widehat{\pi}_{i i}=-\sum_{j=1, j \neq i}^{s} \widehat{\pi}_{i j}$, and $\delta_{i i}=$ $-\sum_{j=1, j \neq i}^{s} \delta_{i j}$. PUTR model (see [15-22]):

$$
\left[\begin{array}{ccccc}
\pi_{11} & ? & \pi_{13} & \cdots & ? \\
? & ? & \pi_{23} & \cdots & \pi_{2 s} \\
\vdots & \vdots & \vdots & \ddots & \vdots \\
? & \pi_{s 2} & ? & \cdots & \pi_{s s}
\end{array}\right]
$$

Obviously, if $U_{k}^{i} \emptyset, \forall i \in \mathbb{S}$, the GUTR model (4) will reduce to the BUTR model (6); if $\delta_{i j}=0, \forall i \in \mathbb{S}, \forall j \in U_{k}^{i}$, the GUTR model (4) will reduce to the PUTR model (7). The GUTR model (4) is more general than the other two models; therefore, it is more practicable. 
For convenience, the system matrices $A_{i} \triangleq A\left(r_{t}=i\right)$, $A_{d i} \triangleq A_{d}\left(r_{t}=i\right)$, and $B_{i} \triangleq B\left(r_{t}=i\right), \Delta B_{i} \triangleq \Delta B\left(r_{t}=i\right), i \in$ $\mathbb{S}$, are known matrix functions of the Markovian process. Then system (1) can be described by

$$
\begin{gathered}
\dot{x}(t)=A_{i} x(t)+A_{d i} x(t-\tau) \\
+\left(B_{i}+\Delta B_{i}\right) q(u(t)), \quad t \geq 0, \\
x_{0}=x(0), \quad r_{0}=r(0) .
\end{gathered}
$$

The following assumptions are assumed to be valid.

Assumption 5. $\Delta B_{i}=B_{i} M_{i} \Xi_{i}(t) F_{i}$ and $\left|M_{i} \Xi_{i}(t) F_{i}\right|_{\infty} \leq \psi_{i}$, where $M_{i}$ and $F_{i}$ are known constant matrices with appropriate dimensions, $\Xi_{i}(t)$ is time-varying uncertain matrix satisfying $\Xi_{i}(t) \Xi_{i}^{T}(t) \leq I$, and parameter $\psi_{i}$ satisfies $0 \leq \psi_{i}<$ 1 .

In addition, the quantizer $q(\cdot)$ is defined by an operator function round (.) which rounds to the nearest integer; that is,

$$
q(u(t))=\mu \cdot \operatorname{round}\left(\frac{u(t)}{\mu}\right)
$$

where $\mu(>0)$ is called a quantizing level of the quantizer. In computer-based control systems, the value of $\mu$ depends on the sampling accuracy and is known a priori. $q(\cdot)$ is the uniform quantizer with the fixed level $\mu$. Define $e_{\mu}=q(u(t))-$ $u(t)$; since each component of $e_{\mu}$ is bounded by the half of the quantizing level $\mu$, we have $\left|e_{\mu}\right|_{\infty} \leq \mu / 2$.

The objective of this paper is to design a state-feedback control law

$$
u(t)=K_{i} x+u_{i c}, \quad K_{i}=K\left(r_{t}\right), \quad \text { when } r_{t}=i
$$

such that the resulting closed-loop system is stochastically stable. The nonlinear part of the controller $u_{i c}$ is designed against the effect of signal quantization, and the linear part $K_{i} x$ is proposed to deal with model uncertainties and unknown transition rates.
Lemma 6 (Petersen, 1987). Given a symmetric matrix $\Pi$ and matrices $M, N$ with appropriate dimensions, then $\Pi+$ $M F(t) N+N^{T} F^{T}(t) M^{T}<0$ for all $F(t)$ satisfying $F^{T}(t) F(t) \leq$ $I$, if and only if there exists a scalar $\varepsilon>0$ such that the following inequality holds:

$$
\Pi+\varepsilon M M^{T}+\varepsilon^{-1} N^{T} N<0 .
$$

Lemma 7. Given any real number $\varepsilon$ and any matrix $Q$, the matrix inequality

$$
\varepsilon\left(Q+Q^{T}\right) \leq \varepsilon^{2} T+Q T^{-1} Q^{T}
$$

holds for any matrix $T>0$.

\section{Stochastic Stability Analysis}

The goal of this section is to develop an analysis result of stability for system (1) with general uncertain TRs.

Theorem 8. Consider that uncertain Markovian jump system (8) with a GUTR matrix (4) is stochastically stable if there exist matrices $P_{i}>0(i \in \mathbb{S}), T_{i j}>0\left(i \notin U_{k}^{i}, j \in U_{k}^{i}\right), V_{i j l}>$ $0\left(i, j \in U_{k}^{i}, U_{u k}^{i} \neq \varnothing, l \in U_{u k}^{i}\right), W_{i j}>0\left(i, j \in U_{k}^{i}, U_{u k}^{i}=\varnothing\right)$, and $Q>0$ such that the following LMIs are feasible for $i=$ $1,2, \ldots, s$.

If $i \notin U_{k}^{i}$,

$$
\begin{gathered}
{\left[\begin{array}{ccccc}
\Gamma_{11} & P_{i} A_{d i} & P_{i k_{1}^{i}}-P_{i} & \cdots & P_{i k_{m}^{i}}-P_{i} \\
* & -Q & 0 & \cdots & 0 \\
* & * & -T_{i k_{1}^{i}} & \cdots & 0 \\
* & * & * & \ddots & \vdots \\
* & * & * & * & -T_{i k_{m}^{i}}
\end{array}\right]<0} \\
\quad P_{i}-P_{j} \geq 0, \quad \forall j \in U_{u k}^{i}, \quad j \neq i .
\end{gathered}
$$

If $i \in U_{k}^{i}$ and $U_{u k}^{i} \neq \varnothing$, for one $l \in U_{u k}^{i}$,

$$
\left[\begin{array}{ccccc}
\Theta_{11} & P_{i} A_{d i} & P_{k_{1}^{i}}-P_{l} & \cdots & P_{k_{m}^{i}}-P_{l} \\
* & -Q & 0 & \cdots & 0 \\
* & * & -V_{i k_{1}^{i} l} & \cdots & 0 \\
* & * & * & \ddots & \vdots \\
* & * & * & * & -V_{i k_{m}^{i} l}
\end{array}\right]<0 .
$$

If $i \in U_{k}^{i}$ and $U_{u k}^{i}=\varnothing$,

$$
\left[\begin{array}{cccccccc}
\Psi_{11} & P_{i} A_{d i} & P_{1}-P_{i} & \cdots & P_{i-1}-P_{i} & P_{i+1}-P_{i} & \cdots & P_{s}-P_{i} \\
* & -Q & 0 & \cdots & 0 & 0 & \cdots & 0 \\
* & * & -W_{i 1} & \cdots & 0 & 0 & \cdots & 0 \\
* & * & * & \ddots & \vdots & \vdots & \vdots & \vdots \\
* & * & * & * & -W_{i(i-1)} & 0 & \vdots & \vdots \\
* & * & * & * & * & -W_{i(i+1)} & \vdots & \vdots \\
* & * & * & * & * & * & \ddots & \vdots \\
* & * & * & * & * & * & * & -W_{i s}
\end{array}\right]<0,
$$


where

$$
\begin{aligned}
\Gamma_{11}= & A_{i}^{T} P_{i}+P_{i} A_{i}+K_{i}^{T} B_{i}^{T} P_{i}+P_{i} B_{i} K_{i}+\sigma_{i} P_{i} B_{i} M_{i} M_{i}^{T} B_{i}^{T} P_{i} \\
& +\frac{1}{\sigma_{i}} K_{i}^{T} F_{i}^{T} F_{i} K_{i}+\sum_{j \in U_{k}^{i}} \widehat{\pi}_{i j}\left(P_{j}-P_{i}\right)+\sum_{j \in U_{k}^{i}} \frac{\delta_{i j}^{2}}{4} T_{i j}+Q \\
\Theta_{11}= & A_{i}^{T} P_{i}+P_{i} A_{i}+K_{i}^{T} B_{i}^{T} P_{i}+P_{i} B_{i} K_{i} \\
& +\sigma_{i} P_{i} B_{i} M_{i} M_{i}^{T} B_{i}^{T} P_{i}+\frac{1}{\sigma_{i}} K_{i}^{T} F_{i}^{T} F_{i} K_{i} \\
& +\sum_{j \in U_{k}^{i}} \widehat{\pi}_{i j}\left(P_{j}-P_{l}\right)+\sum_{j \in U_{k}^{i}} \frac{\delta_{i j}^{2}}{4} V_{i j l}+Q \\
\Psi_{11}= & A_{i}^{T} P_{i}+P_{i} A_{i}+K_{i}^{T} B_{i}^{T} P_{i}+P_{i} B_{i} K_{i} \\
& +\sigma_{i} P_{i} B_{i} M_{i} M_{i}^{T} B_{i}^{T} P_{i}+\frac{1}{\sigma_{i}} K_{i}^{T} F_{i}^{T} F_{i} K_{i} \\
& +\sum_{j \in \mathbb{S}, j \neq i} \widehat{\pi}_{i j}\left(P_{j}-P_{i}\right)+\sum_{j \in \mathbb{S}, j \neq i} \frac{\delta_{i j}^{2}}{4} W_{i j}+Q .
\end{aligned}
$$

Then the controller designed as

$$
u(t)=K_{i} x+u_{i c}
$$

can drive the state trajectory to the origin asymptotically, where $K_{i}=Y_{i} X_{i}^{-1}, P_{i}=X_{i}^{-1}$, and $u_{i c}=(1+\psi) u /(2(1-$ $\psi)) \operatorname{sign}\left(x^{T} P_{i} B_{i}\right)$.

Proof. Take the Lyapunov function candidate $V=x^{T} P_{i} x+$ $\int_{t-\tau}^{t} x^{T}(\alpha) Q x(\alpha) d \alpha$; then along the system trajectory of plant $(8)$, the weak infinitesimal operator $\mathfrak{\Im}_{a}^{x}[\cdot]$ of the process $x(t), r_{t}, t \geq 0$, for plant (8) at the point $t, x, i$ is as follows:

$$
\begin{aligned}
\mathfrak{\mho}_{a}^{x}[V]= & \dot{x}^{T} P_{i} x+x^{T} P_{i} \dot{x}+x^{T} \sum_{j=1}^{\mathbb{N}} \lambda_{i j} P_{j} x \\
= & {\left[A_{i} x+A_{d i} x(t-\tau)\right.} \\
& \left.+\left(B_{i}+\Delta B_{i}\right)\left(K_{i} x+u_{i c}+e_{\mu}\right)\right]^{T} P_{i} x \\
& +x^{T} P_{i}\left[A_{i} x+A_{d i} x(t-\tau)\right. \\
& \left.+\left(B_{i}+\Delta B_{i}\right)\left(K_{i} x+u_{i c}+e_{\mu}\right)\right] \\
& +x^{T} \sum_{j=1}^{\mathbb{N}} \lambda_{i j} P_{j} x \\
= & x^{T}\left[A_{i}^{T} P_{i}+P_{i} A_{i}+P_{i}\left(B_{i}+\Delta B_{i}\right) K_{i}\right.
\end{aligned}
$$

$$
\begin{gathered}
\left.+K_{i}^{T}\left(B_{i}+\Delta B_{i}\right)^{T} P_{i}+Q\right] x \\
+x^{T}(t-\tau) A_{d i}^{T} P_{i} x+x^{T} P_{i} A_{d i} x(t-\tau) \\
-x^{T}(t-\tau) Q x(t-\tau) \\
+2 x^{T} P_{i}\left(B_{i}+\Delta B_{i}\right)\left(u_{i c}+e_{\mu}\right)+x^{T} \sum_{j=1}^{\mathbb{N}} \lambda_{i j} P_{j} x .
\end{gathered}
$$

According to Assumption 2, $u_{i c}$ in Theorem 8, and Lemma 6, one can obtain that

$$
\begin{aligned}
& 2 x^{T} P_{i}\left(B_{i}+\Delta B_{i}\right)\left(u_{i c}+e_{\mu}\right) \\
&=2 x^{T} P_{i} B_{i} u_{i c}+2 x^{T} P_{i} \Delta B_{i}\left(u_{i c}+e_{\mu}\right)+2 x^{T} P_{i} B_{i} e_{\mu} \\
&=2 x^{T} P_{i} B_{i} u_{i c}+2 x^{T} P_{i} B_{i} M_{i} \Xi_{i}(t) F_{i}\left(u_{i c}+e_{\mu}\right)+2 x^{T} P_{i} B_{i} e_{\mu} \\
& \leq 2 x^{T} P_{i} B_{i} u_{i c}+2\left|x^{T} P_{i} B_{i}\right|_{1}\left|M_{i} \Xi_{i}(t) F_{i}\right|_{\infty}\left(\left|u_{i c}\right|_{\infty}+\frac{\mu}{2}\right) \\
&+2\left|x^{T} P_{i} B_{i}\right|_{1} \frac{\mu}{2} \\
&= 2 x^{T} P_{i} B_{i} u_{i c}+2\left|x^{T} P_{i} B_{i} B_{i}\right|_{1} \psi\left(\left|u_{i c}\right|_{\infty}+\frac{\mu}{2}\right) \\
&+\left|x^{T} P_{i} B_{i}\right|_{1} \mu \\
& \leq 2 x^{T} P_{i} B_{i} u_{i c}+(1+\psi)\left|x^{T} P_{i} B_{i}\right|_{1} \mu \\
&+2 \psi\left|x^{T} P_{i} B_{i}\right|_{1}\left|u_{i c}\right|_{\infty} \\
&+\frac{(1+\psi) \mu}{1-\psi}\left|x^{T} P_{i} B_{i}\right|_{1}+(1+\psi)\left|x^{T} P_{i} B_{i}\right|_{1} \mu \\
&+\frac{\psi(1+\psi) \mu}{1-\psi}\left|x^{T} P_{i} B_{i}\right|_{1}=0 . \\
&= \\
& 1+
\end{aligned}
$$

It follows from (19) and (20) that

$$
\begin{aligned}
\mathfrak{\Im}_{a}^{x}[V] \leq & x^{T}\left[A_{i}^{T} P_{i}+P_{i} A_{i}+P_{i}\left(B_{i}+\Delta B_{i}\right) K_{i}\right. \\
& \left.+K_{i}^{T}\left(B_{i}+\Delta B_{i}\right)^{T} P_{i}+Q\right] x \\
& +x^{T}(t-\tau) A_{d i}^{T} P_{i} x+x^{T} P_{i} A_{d i} x(t-\tau) \\
& -x^{T}(t-\tau) Q x(t-\tau)+x^{T} \sum_{j=1}^{\mathbb{N}} \lambda_{i j} P_{j} x
\end{aligned}
$$

Since $\Delta B_{i}=B_{i} M_{i} \Xi_{i}(t) F_{i}$, we introduce

$$
\begin{aligned}
\Phi_{i} \triangleq & x^{T}\left[A_{i}^{T} P_{i}+P_{i} A_{i}+K_{i}^{T} B_{i}^{T} P_{i}+P_{i} B_{i} K_{i}\right. \\
& \left.\quad+P_{i} B_{i} M_{i} \Xi_{i}(t) F_{i} K_{i}+K_{i}^{T} F_{i}^{T} \Xi_{i}^{T}(t) M_{i}^{T} B_{i}^{T} P_{i}+Q\right] x \\
& +x^{T}(t-\tau) A_{d i}^{T} P_{i} x+x^{T} P_{i} A_{d i} x(t-\tau) \\
& -x^{T}(t-\tau) Q x(t-\tau) .
\end{aligned}
$$


Then, the above inequality can be rewritten as

$$
\mathfrak{\mho}_{a}^{x}[V] \leq \Phi_{i}+x^{T}\left[\sum_{j=1}^{\mathbb{N}} \lambda_{i j} P_{j}\right] x
$$

Three cases should be considered.

Case I $\left(i \notin U_{k}^{i}\right)$. In this case, note that $\sum_{j \in U_{u k}^{i}, j \neq i} \pi_{i j}=-\pi_{i i}-$ $\sum_{j \in U_{k}^{i}} \pi_{i j}$ and $\pi_{i j} \geq 0, j \in U_{u k}^{i}, j \neq i$; then from (23), we have

$$
\begin{aligned}
\mathfrak{\Im}_{a}^{x}[V] \leq \Phi_{i}+x^{T}\left[\sum_{j \in U_{k}^{i}} \pi_{i j} P_{j}+\pi_{i i} P_{i}+\sum_{j \in U_{u k}^{i}, j \neq i} \pi_{i j} P_{i}\right] x \\
=\Phi_{i}+x^{T}\left[\sum_{j \in U_{k}^{i}} \pi_{i j} P_{j}+\pi_{i i} P_{i}\right. \\
\left.+\left(-\pi_{i i}-\sum_{j \in U_{k}^{i}} \pi_{i j}\right) P_{i}\right] x \\
=\Phi_{i}+x^{T}\left[\sum_{j \in U_{k}^{i}} \pi_{i j}\left(P_{j}-P_{i}\right)\right] x \\
=\Phi_{i}+x^{T}\left[\sum_{j \in U_{k}^{i}}\left(\hat{\pi}_{i j}+\Delta_{i j}\right)\left(P_{j}-P_{i}\right)\right] x \\
=\Phi_{i}+x^{T}\left[\sum_{j \in U_{k}^{i}} \widehat{\pi}_{i j}\left(P_{j}-P_{i}\right)+\sum_{j \in U_{k}^{i}} \Delta_{i j}\left(P_{j}-P_{i}\right)\right] x .
\end{aligned}
$$

There, note that $\pi_{i j}=\widehat{\pi}_{i j}+\Delta_{i j}$ for $j \in U_{k}^{i}$.

On the other hand, in view of Lemma 7, we have

$$
\begin{aligned}
& \sum_{j \in U_{k}^{i}} \Delta_{i j}\left(P_{j}-P_{i}\right) \\
& \quad=\sum_{j \in U_{k}^{i}}\left[\frac{1}{2} \Delta_{i j}\left(P_{j}-P_{i}\right)+\frac{1}{2} \Delta_{i j}\left(P_{j}-P_{i}\right)\right] \\
& \leq \sum_{j \in U_{k}^{i}}\left[\left(\frac{1}{2} \Delta_{i j}\right)^{2} T_{i j}+\left(P_{j}-P_{i}\right) T_{i j}^{-1}\left(P_{j}-P_{i}\right)\right] \\
& \leq \sum_{j \in U_{k}^{i}}\left[\frac{\delta_{i j}^{2}}{4} T_{i j}+\left(P_{j}-P_{i}\right) T_{i j}^{-1}\left(P_{j}-P_{i}\right)\right] .
\end{aligned}
$$

From (24) and (25), we have

$$
\begin{aligned}
\mathfrak{\mho}_{a}^{x}[V] \leq & \Phi_{i}+x^{T} \\
& \times\left\{\sum_{j \in U_{k}^{i}} \hat{\pi}_{i j}\left(P_{j}-P_{i}\right)\right. \\
& \left.+\sum_{j \in U_{k}^{i}}\left[\frac{\delta_{i j}^{2}}{4} T_{i j}+\left(P_{j}-P_{i}\right) T_{i j}^{-1}\left(P_{j}-P_{i}\right)\right]\right\} x .
\end{aligned}
$$

Hence, $\mathfrak{\Im}_{a}^{x}[V]<0$ holds if

$$
\mathscr{H}=\left[\begin{array}{cc}
\mathscr{A}_{11} & P_{i} A_{d i} \\
* & -Q
\end{array}\right]<0,
$$

where

$$
\begin{aligned}
\mathscr{A}_{11}= & A_{i}^{T} P_{i}+P_{i} A_{i}+K_{i}^{T} B_{i}^{T} P_{i}+P_{i} B_{i} K_{i}+P_{i} B_{i} M_{i} \Xi_{i}(t) F_{i} K_{i} \\
& +K_{i}^{T} F_{i}^{T} \Xi_{i}^{T}(t) M_{i}^{T} B_{i}^{T} P_{i}+Q+\sum_{j \in U_{k}^{i}} \widehat{\pi}_{i j}\left(P_{j}-P_{i}\right) \\
& +\sum_{j \in U_{k}^{i}}\left[\frac{\delta_{i j}^{2}}{4} T_{i j}+\left(P_{j}-P_{i}\right) T_{i j}^{-1}\left(P_{j}-P_{i}\right)\right] .
\end{aligned}
$$

Let

$$
\begin{aligned}
\overline{\mathscr{A}}_{11}= & A_{i}^{T} P_{i}+P_{i} A_{i}+K_{i}^{T} B_{i}^{T} P_{i}+P_{i} B_{i} K_{i}+Q \\
& +\sum_{j \in U_{k}^{i}} \hat{\pi}_{i j}\left(P_{j}-P_{i}\right) \\
& +\sum_{j \in U_{k}^{i}}\left[\frac{\delta_{i j}^{2}}{4} T_{i j}+\left(P_{j}-P_{i}\right) T_{i j}^{-1}\left(P_{j}-P_{i}\right)\right] ;
\end{aligned}
$$

then we have

$$
\begin{aligned}
\mathscr{H}= & {\left[\begin{array}{cc}
\bar{A}_{11} & P_{i} A_{d i} \\
* & -Q
\end{array}\right]+\left[\begin{array}{c}
P_{i} B_{i} M_{i} \\
0
\end{array}\right] \Xi_{i}(t)\left[F_{i} K_{i} 0\right] } \\
& +\left[\begin{array}{ll}
F_{i} K_{i} & 0
\end{array}\right]^{T} \Xi_{i}^{T}(t)\left[\begin{array}{c}
P_{i} B_{i} M_{i} \\
0
\end{array}\right]^{T}<0,
\end{aligned}
$$

which is equivalent to (13) by Schur complement.

Case II $\left(i \in U_{k}^{i}\right.$ and $\left.U_{u k}^{i} \neq \varnothing\right)$. There must be an $l \in U_{u k}^{i}$ such that $P(l)-P(j) \geq 0, \forall j \in U_{u k}^{i}$. We define

$$
\begin{aligned}
\mathfrak{\Im}_{a}^{x}[V] & \leq \Phi_{i}+x^{T}\left[\sum_{j \in U_{k}^{i}} \lambda_{i j} P(j)+\sum_{j \in U_{u k}^{i}} \lambda_{i j} P(l)\right] x \\
& =\Phi_{i}+x^{T}\left[\sum_{j \in U_{k}^{i}} \pi_{i j} P_{j}-\left(\sum_{j \in U_{k}^{i}} \pi_{i j}\right) P_{l}\right] x
\end{aligned}
$$




$$
\begin{aligned}
& =\Phi_{i}+x^{T}\left[\sum_{j \in U_{k}^{i}} \pi_{i j}\left(P_{j}-P_{l}\right)\right] x \\
& =\Phi_{i}+x^{T}\left[\sum_{j \in U_{k}^{i}}\left(\hat{\pi}_{i j}+\Delta_{i j}\right)\left(P_{j}-P_{l}\right)\right] x \\
& =\Phi_{i}+x^{T}\left[\sum_{j \in U_{k}^{i}} \hat{\pi}_{i j}\left(P_{j}-P_{l}\right)+\sum_{j \in U_{k}^{i}} \Delta_{i j}\left(P_{j}-P_{l}\right)\right] x .
\end{aligned}
$$

By using Lemma 7 again, we have

$$
\begin{aligned}
& \sum_{j \in U_{k}^{i}} \Delta_{i j}\left(P_{j}-P_{l}\right) \\
& =\sum_{j \in U_{k}^{i}}\left[\frac{1}{2} \Delta_{i j}\left(P_{j}-P_{l}\right)+\frac{1}{2} \Delta_{i j}\left(P_{j}-P_{l}\right)\right] \\
& \leq \sum_{j \in U_{k}^{i}}\left[\left(\frac{1}{2} \Delta_{i j}\right)^{2} V_{i j l}+\left(P_{j}-P_{l}\right) V_{i j l}^{-1}\left(P_{j}-P_{l}\right)\right] \\
& \leq \sum_{j \in U_{k}^{i}}\left[\frac{\delta_{i j}^{2}}{4} V_{i j l}+\left(P_{j}-P_{l}\right) V_{i j l}^{-1}\left(P_{j}-P_{l}\right)\right]
\end{aligned}
$$

From (31) and (32), we have

$$
\begin{aligned}
\mathfrak{\Im}_{a}^{x}[V] \leq & \Phi_{i}+x^{T} \\
& \times\left\{\sum_{j \in U_{k}^{i}} \hat{\pi}_{i j}\left(P_{j}-P_{l}\right)\right. \\
& \left.+\sum_{j \in U_{k}^{i}}\left[\frac{\delta_{i j}^{2}}{4} V_{i j l}+\left(P_{j}-P_{l}\right) V_{i j l}^{-1}\left(P_{j}-P_{l}\right)\right]\right\} x .
\end{aligned}
$$

Hence, $\mathfrak{\Im}_{a}^{x}[V]<0$ holds if

$$
\mathfrak{A}=\left[\begin{array}{cc}
\mathfrak{A}_{11} & P_{i} A_{d i} \\
* & -Q
\end{array}\right]<0,
$$

where

$$
\begin{aligned}
\mathfrak{A}_{11}= & A_{i}^{T} P_{i}+P_{i} A_{i}+K_{i}^{T} B_{i}^{T} P_{i}+P_{i} B_{i} K_{i}+P_{i} B_{i} M_{i} \Xi_{i}(t) F_{i} K_{i} \\
& +K_{i}^{T} F_{i}^{T} \Xi_{i}^{T}(t) M_{i}^{T} B_{i}^{T} P_{i}+Q+\sum_{j \in U_{k}^{i}} \widehat{\pi}_{i j}\left(P_{j}-P_{l}\right) \\
& +\sum_{j \in U_{k}^{i}}\left[\frac{\delta_{i j}^{2}}{4} V_{i j l}+\left(P_{j}-P_{l}\right) V_{i j l}^{-1}\left(P_{j}-P_{l}\right)\right] .
\end{aligned}
$$

Let

$$
\begin{aligned}
\overline{\mathfrak{A}}_{11}= & A_{i}^{T} P_{i}+P_{i} A_{i}+K_{i}^{T} B_{i}^{T} P_{i}+P_{i} B_{i} K_{i}+Q \\
& +\sum_{j \in U_{k}^{i}} \widehat{\pi}_{i j}\left(P_{j}-P_{l}\right) \\
& +\sum_{j \in U_{k}^{i}}\left[\frac{\delta_{i j}^{2}}{4} V_{i j l}+\left(P_{j}-P_{l}\right) V_{i j l}^{-1}\left(P_{j}-P_{l}\right)\right] ;
\end{aligned}
$$

then we have

$$
\begin{aligned}
\mathfrak{U}= & {\left[\begin{array}{cc}
\overline{\mathfrak{A}}_{11} & P_{i} A_{d i} \\
* & -Q
\end{array}\right]+\left[\begin{array}{c}
P_{i} B_{i} M_{i} \\
0
\end{array}\right] \Xi_{i}(t)\left[F_{i} K_{i} 0\right] } \\
& +\left[\begin{array}{ll}
F_{i} K_{i} & 0
\end{array}\right]^{T} \Xi_{i}^{T}(t)\left[\begin{array}{c}
P_{i} B_{i} M_{i} \\
0
\end{array}\right]^{T}<0,
\end{aligned}
$$

which is equivalent to (14) by Schur complement.

Case III $\left(i \in U_{k}^{i}\right.$ and $\left.U_{u k}^{i}=\varnothing\right)$. In this case,

$$
\begin{aligned}
\mathfrak{\Im}_{a}^{x}[V] & \leq \Phi_{i}+x^{T}\left[\sum_{j=1, j \neq i}^{N} \lambda_{i j} P(j)+\lambda_{i i} P(i)\right] x \\
& =\Phi_{i}+x^{T}\left[\sum_{j \in U_{k}^{i}} \pi_{i j} P_{j}-\left(\sum_{j \in U_{k}^{i}} \pi_{i j}\right) P_{i}\right] x \\
& =\Phi_{i}+x^{T}\left[\sum_{j \in U_{k}^{i}} \pi_{i j}\left(P_{j}-P_{i}\right)\right] x \\
& =\Phi_{i}+x^{T}\left[\sum_{j \in U_{k}^{i}}\left(\hat{\pi}_{i j}+\Delta_{i j}\right)\left(P_{j}-P_{i}\right)\right] x \\
& =\Phi_{i}+x^{T}\left[\sum_{j \in U_{k}^{i}} \hat{\pi}_{i j}\left(P_{j}-P_{i}\right)+\sum_{j \in U_{k}^{i}} \Delta_{i j}\left(P_{j}-P_{i}\right)\right] x .
\end{aligned}
$$

In view of Lemma 7, we have

$$
\begin{aligned}
& \sum_{j=1, j \neq i}^{s} \Delta_{i j}\left(P_{j}-P_{i}\right) \\
& \quad=\sum_{j=1, j \neq i}^{s}\left[\frac{1}{2} \Delta_{i j}\left(P_{j}-P_{i}\right)+\frac{1}{2} \Delta_{i j}\left(P_{j}-P_{i}\right)\right] \\
& \leq \sum_{j=1, j \neq i}^{s}\left[\left(\frac{1}{2} \Delta_{i j}\right)^{2} W_{i j}+\left(P_{j}-P_{i}\right) W_{i j}^{-1}\left(P_{j}-P_{i}\right)\right] \\
& \quad \leq \sum_{j=1, j \neq i}^{s}\left[\frac{\delta_{i j}^{2}}{4} W_{i j}+\left(P_{j}-P_{i}\right) W_{i j}^{-1}\left(P_{j}-P_{i}\right)\right] .
\end{aligned}
$$


From (38) and (39), we have

$$
\begin{aligned}
& \mathfrak{\mho}_{a}^{x}[V] \leq \Phi_{i}+x^{T} \\
& \times\left\{\sum_{j=1, j \neq i}^{s} \hat{\pi}_{i j}\left(P_{j}-P_{i}\right)\right. \\
&+\sum_{j=1, j \neq i}^{s}\left[\frac{\delta_{i j}^{2}}{4} W_{i j}+\left(P_{j}-P_{i}\right)\right. \\
&\left.\left.\times W_{i j}^{-1}\left(P_{j}-P_{i}\right)\right]\right\} x .
\end{aligned}
$$

Hence, $\mathfrak{\Im}_{a}^{x}[V]<0$ holds if

$$
\mathscr{R}=\left[\begin{array}{cc}
\mathscr{R}_{11} & P_{i} A_{d i} \\
* & -Q
\end{array}\right]<0,
$$

where

$$
\begin{aligned}
\mathscr{R}_{11}= & A_{i}^{T} P_{i}+P_{i} A_{i}+K_{i}^{T} B_{i}^{T} P_{i}+P_{i} B_{i} K_{i}+P_{i} B_{i} M_{i} \Xi_{i}(t) F_{i} K_{i} \\
& +K_{i}^{T} F_{i}^{T} \Xi_{i}^{T}(t) M_{i}^{T} B_{i}^{T} P_{i}+Q+\sum_{j \in U_{k}^{i}} \widehat{\pi}_{i j}\left(P_{j}-P_{i}\right) \\
& +\sum_{j \in U_{k}^{i}}\left[\frac{\delta_{i j}^{2}}{4} W_{i j}+\left(P_{j}-P_{i}\right) W_{i j}^{-1}\left(P_{j}-P_{i}\right)\right] .
\end{aligned}
$$

Let

$$
\begin{aligned}
\overline{\mathscr{R}}_{11}= & A_{i}^{T} P_{i}+P_{i} A_{i}+K_{i}^{T} B_{i}^{T} P_{i}+P_{i} B_{i} K_{i}+Q \\
& +\sum_{j \in U_{k}^{i}} \hat{\pi}_{i j}\left(P_{j}-P_{i}\right) \\
& +\sum_{j \in U_{k}^{i}}\left[\frac{\delta_{i j}^{2}}{4} W_{i j}+\left(P_{j}-P_{i}\right) W_{i j}^{-1}\left(P_{j}-P_{i}\right)\right] ;
\end{aligned}
$$

then we have

$$
\begin{aligned}
\mathscr{R}= & {\left[\begin{array}{cc}
\overline{\mathscr{R}}_{11} & P_{i} A_{d i} \\
* & -Q
\end{array}\right]+\left[\begin{array}{c}
P_{i} B_{i} M_{i} \\
0
\end{array}\right] \Xi_{i}(t)\left[\begin{array}{ll}
F_{i} K_{i} & 0
\end{array}\right] } \\
& +\left[\begin{array}{ll}
F_{i} K_{i} & 0
\end{array}\right]^{T} \Xi_{i}^{T}(t)\left[\begin{array}{c}
P_{i} B_{i} M_{i} \\
0
\end{array}\right]^{T}<0,
\end{aligned}
$$

which is equivalent to (15) by Schur complement. The proof is completed.

Remark 9. As mentioned in Section 2, if, $U_{u k}^{i}=\varnothing \forall i \in \mathbb{S}$, then GUTR matrix reduces to BUTR one. Similarly, if, $\delta_{i j}=$ $0 \forall j \in U_{k}^{i}, \forall i \in \mathbb{S}$, GUTR matrix reduces to PUTR one. Therefore, Theorem 8 can also be applicable to the MJSs with BUTRs or PUTRs. Because the BUTR methods require the estimate of every rate to be known, such methods cannot be applied to the GUTR model. By replacing the uncertain TRs with unknown ones, the generally uncertain TR matrix can become partly known TR matrix, so that the PUTR methods can be applied to GUTR model. However, such methods are inevitably conservative for GUTRs since the information of the TRs' estimates cannot be utilized.

\section{Conclusions}

The stability problems for a class of Markovian jump linear systems with generally uncertain transition rates are investigated in this paper. The considered systems are more general than the systems with bounded uncertain transition rates or partly unknown transition rates, which can be viewed as two special cases of the systems we tackled here. The LMI-based stochastic stability condition for the underlying systems is derived. There are some possible directions to extend the proposed model and method. Tracking control and faulttolerant control are two important research areas due to their wide application in the practical systems [40-46]. However, for the tracking control and fault-tolerant control, no research has focused on the case of general uncertain transition rates. Therefore, it is worth further extending the proposed method to deal with these problems.

\section{Conflict of Interests}

The authors declare that there is no conflict of interests regarding the publication of this paper.

\section{Acknowledgments}

The authors would like to thank the editors and the anonymous reviewers for their valuable comments and constructive suggestions. This research is supported by the Natural Science Foundation of Guangxi Autonomous Region (nos. 2012GXNSFBA053003, 2013YB141), the National Natural Science Foundations of China (60973048, 61272077, 60974025, 60673101, and 60939003), National 863 Plan Project (2008AA04Z401, 2009AA043404), the Natural Science Foundation of Shandong Province (no. Y2007G30), the Scientific and Technological Project of Shandong Province (no. 2007GG3WZ04016), the Science Foundation of Harbin Institute of Technology (Weihai) (HIT(WH) 200807), the Natural Scientific Research Innovation Foundation in Harbin Institute of Technology (HIT.NSRIF. 2001120), and Shandong Provincial Key Laboratory of Industrialy.

\section{References}

[1] X. Mao and C. Yuan, Stochastic Differential Equations with Markovian Switching, Imperial College Press, 2006.

[2] H. Zhang, Y. Shi, and J. M. Wang, “On energy-to-peak filtering for nonuniformly sampled nonlinear sampled nonlinear systems: a Markovian jump system approach," IEEE Transactions on Fuzzy Systems, vol. 22, pp. 212-222, 2014.

[3] Z. Wu, P. Shi, H. Su, and J. Chu, "Stochastic synchronization of Markovian jump neural networks with time-varying delay 
using sampled-data," IEEE Transactions on Cybernetics, vol. 43, pp. 1796-1806, 2013.

[4] Z. D. Wang, Y. R. Liu, L. Yu, and X. H. Liu, "Exponential stability of delayed recurrent neural networks with Markovian jumping parameters," Physics Letters A, vol. 356, pp. 346-352, 2006.

[5] Z.-G. Wu, J. H. Park, H. Su, and J. Chu, "Stochastic stability analysis for discrete-time singular Markov jump systems with time-varying delay and piecewise-constant transition probabilities," Journal of the Franklin Institute. Engineering and Applied Mathematics, vol. 349, no. 9, pp. 2889-2902, 2012.

[6] Z. Wu, H. Su, and J. Chu, " $H_{\infty}$ model reduction for discrete singular Markovian jump systems," Proceedings of the Institution of Mechanical Engineers, vol. 223, no. 7, pp. 1017-1025, 2009.

[7] M. Liu, P. Shi, L. Zhang, and X. Zhao, "Fault-tolerant control for nonlinear Markovian jump systems via proportional and derivative sliding mode observer technique," IEEE Transactions on Circuits and Systems I, vol. 58, no. 11, pp. 2755-2764, 2011.

[8] Y. Kao, C. Wang, and L. Zhang, "Delay-dependent exponential stability of impulsive Markovian jumping cohen-grossberg neural networks with reaction-diffusion and mixed delays," Neural Processing Letters, vol. 38, no. 3, pp. 321-346, 2013.

[9] Y.-G. Kao, J.-F. Guo, C.-H. Wang, and X.-Q. Sun, "Delaydependent robust exponential stability of Markovian jumping reaction-diffusion Cohen-Grossberg neural networks with mixed delays," Journal of the Franklin Institute. Engineering and Applied Mathematics, vol. 349, no. 6, pp. 1972-1988, 2012.

[10] Y. Kao, C. Wang, F. Zha, and H. Cao, "Stability in mean of partial variables for stochastic reaction-diffusion systems with Markovian switching," Journal of the Franklin Institute. Engineering and Applied Mathematics, vol. 351, no. 1, pp. 500$512,2014$.

[11] H. Zhang, Y. Shi, and J. Wang, “Observer-based tracking controller design for networked predictive control systems with uncertain Markov delays," International Journal of Control, vol. 86, no. 10, pp. 1824-1836, 2013.

[12] M. Karan, P. Shi, and C. Y. Kaya, "Transition probability bounds for the stochastic stability robustness of continuousand discrete-time Markovian jump linear systems," Automatica, vol. 42, no. 12, pp. 2159-2168, 2006.

[13] J. Xiong and J. Lam, "Robust $H_{2}$ control of Markovian jump systems with uncertain switching probabilities," International Journal of Systems Science. Principles and Applications of Systems and Integration, vol. 40, no. 3, pp. 255-265, 2009.

[14] J. Xiong, J. Lam, H. Gao, and D. W. C. Ho, "On robust stabilization of Markovian jump systems with uncertain switching probabilities," Automatica, vol. 41, no. 5, pp. 897-903, 2005.

[15] L. Zhang and E.-K. Boukas, "Stability and stabilization of Markovian jump linear systems with partly unknown transition probabilities," Automatica, vol. 45, no. 2, pp. 463-468, 2009.

[16] L. Zhang and E.-K. Boukas, "Mode-dependent $H_{\infty}$ filtering for discrete-time Markovian jump linear systems with partly unknown transition probabilities," Automatica, vol. 45 , no. 6 , pp. 1462-1467, 2009.

[17] L. Zhang and E.-K. Boukas, " $H_{\infty}$ control for discrete-time Markovian jump linear systems with partly unknown transition probabilities," International Journal of Robust and Nonlinear Control, vol. 19, no. 8, pp. 868-883, 2009.

[18] L. Zhang, E.-K. Boukas, and J. Lam, "Analysis and synthesis of Markov jump linear systems with time-varying delays and partially known transition probabilities," IEEE Transactions on Automatic Control, vol. 53, no. 10, pp. 2458-2464, 2008.
[19] H. Zhang, J. Wang, and Y. Shi, "Robust $H_{\infty}$ sliding-mode control for Markovian jump systems subject to intermittent observations and partially known transition probabilities," Systems \& Control Letters, vol. 62, no. 12, pp. 1114-1124, 2013.

[20] Y. Zhang, Y. He, M. Wu, and J. Zhang, "Stabilization for Markovian jump systems with partial information on transition probability based on free-connection weighting matrices," Automatica, vol. 47, no. 1, pp. 79-84, 2011.

[21] L. Xiong, J. Tian, and X. Liu, "Stability analysis for neutral Markovian jump systems with partially unknown transition probabilities," Journal of the Franklin Institute. Engineering and Applied Mathematics, vol. 349, no. 6, pp. 2193-2214, 2012.

[22] Y. Kao, W. Changhong, X. Jing, and K. Hamid Reza, "Stabilisation of singular Markovian jump systems with generally uncertain transition rates," IEEE Transactions on Automatic Control, 2014.

[23] Y. Guo and Z. Wang, "Stability of Markovian jump systems with generally uncertain transition rates," Journal of the Franklin Institute. Engineering and Applied Mathematics, vol. 350, no. 9, pp. 2826-2836, 2013.

[24] B.-C. Zheng and G.-H. Yang, "Quantised feedback stabilisation of planar systems via switching-based sliding-mode control," IET Control Theory \& Applications, vol. 6, no. 1, pp. 149-156, 2012.

[25] D. Liberzon, "Hybrid feedback stabilization of systems with quantized signals," Automatica, vol. 39, no. 9, pp. 1543-1554, 2003.

[26] R. W. Brockett and D. Liberzon, "Quantized feedback stabilization of linear systems," IEEE Transactions on Automatic Control, vol. 45, no. 7, pp. 1279-1289, 2000.

[27] B.-C. Zheng and G.-H. Yang, "Decentralized sliding mode quantized feedback control for a class of uncertain large-scale systems with dead-zone input," Nonlinear Dynamics, vol. 71, no. 3, pp. 417-427, 2013.

[28] M. Fu and L. Xie, "The sector bound approach to quantized feedback control," IEEE Transactions on Automatic Control, vol. 50, no. 11, pp. 1698-1711, 2005.

[29] M. Fu and L. Xie, "Quantized feedback control for linear uncertain systems," International Journal of Robust and Nonlinear Control, vol. 20, no. 8, pp. 843-857, 2010.

[30] E. Tian, D. Yue, and X. Zhao, "Quantised control design for networked systems," IET Control Theory and Applications, vol. 1, no. 6, pp. 1693-1699, 2007.

[31] S. W. Yun, Y. J. Choi, and P. Park, " $H_{2}$ control of continuous-time uncertain linear systems with input quantization and matched disturbances," Automatica, vol. 45, no. 10, pp. 2435-2439, 2009.

[32] W.-W. Che and G.-H. Yang, "Quantised $H_{\infty}$ filter design for discrete-time systems," International Journal of Control, vol. 82, no. 2, pp. 195-206, 2009.

[33] C. Peng and Y.-C. Tian, "Networked $H_{\infty}$ control of linear systems with state quantization," Information Sciences, vol. 177, no. 24, pp. 5763-5774, 2007.

[34] B.-C. Zheng and G.-H. Yang, "Quantized output feedback stabilization of uncertain systems with input nonlinearities via sliding mode control," International Journal of Robust and Nonlinear Control, vol. 24, no. 2, pp. 228-246, 2014.

[35] B.-C. Zheng and G.-H. Yang, "Robust quantized feedback stabilization of linear systems based on sliding mode control," Optimal Control Applications \& Methods, vol. 34, no. 4, pp. 458471, 2013. 
[36] B. C. Zheng and G. H. Yang, " $H_{2}$ control of linear uncertain systems considering input quantization with encoder/decoder mismatch," ISA Transactions, vol. 52, pp. 557-582, 2013.

[37] N. Xiao, L. Xie, and M. Fu, "Stabilization of Markov jump linear systems using quantized state feedback," Automatica, vol. 46, no. 10, pp. 1696-1702, 2010.

[38] D. Ye, B. C. Zheng, and G. H. Yang, " $H_{2}$ control of Markov jump linear systems with unknown transition rates and input quantization," in Proceedings of the 31st Chinese Control Conference, pp. 2673-2678, 2012.

[39] X.-G. Zhao, D. Ye, and J.-D. Han, " $H_{2}$ control for the continuous-time Markovian jump linear uncertain systems with partly known transition rates and input quantization," Mathematical Problems in Engineering, vol. 2013, Article ID 426271, 9 pages, 2013.

[40] S. Yin, H. Luo, and S. Ding, "Real-time implementation of faulttolerant control systems with performance optimization," IEEE Transactions on Industrial Electronics, vol. 64, no. 5, pp. 24022411, 2014.

[41] S. Yin, G. Wang, and H. Karimi, "Data-driven design of robust fault detection system for wind turbines," Mechatronics, 2013.

[42] S. Yin, S. X. Ding, A. H. A. Sari, and H. Hao, "Data-driven monitoring for stochastic systems and its application on batch process," International Journal of Systems Science. Principles and Applications of Systems and Integration, vol. 44, no. 7, pp. 13661376, 2013.

[43] S. Yin, S. Ding, A. Haghani, H. Hao, and P. Zhang, "A comparison study of basic datadriven fault diagnosis and process monitoring methods on the benchmark Tennessee Eastman process," Journal of Process Control, vol. 22, no. 9, pp. 1567-1581, 2012.

[44] H. Zhang, X. J. Zhang, and J. M. Wang, "Robust gain-scheduling energy-to-peak control of vehicle lateral dynamics stabilization,” Vehicle System Dynamics, vol. 52, pp. 309-340, 2014.

[45] H. Zhang, Y. Shi, and B. X. Mu, "Optimal $H_{\infty}$-based linearquadratic regulator tracking control for discrete-time TakagiSugeno fuzzy systems with preview actions," Journal of Dynamic Systems, Measurement and Control, vol. 135, 5 pages, 2013.

[46] H. Zhang, Y. Shi, and M. X. Liu, " $H_{\infty}$ step tracking control for networked discrete-time nonlinear systems with integral and predictive actions," IEEE Transactions on Industrial Informatics, no. 9, pp. 337-345, 2013. 


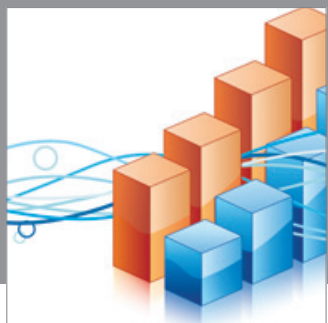

Advances in

Operations Research

mansans

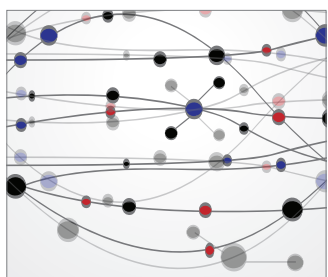

The Scientific World Journal
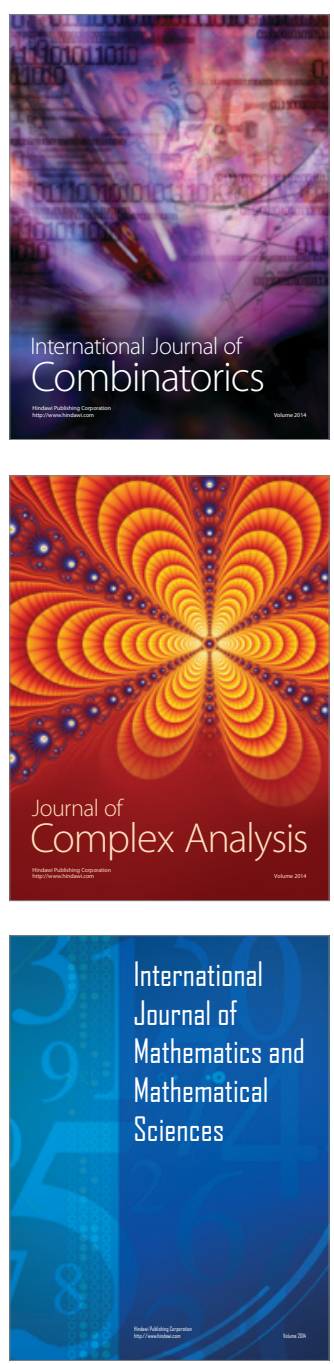
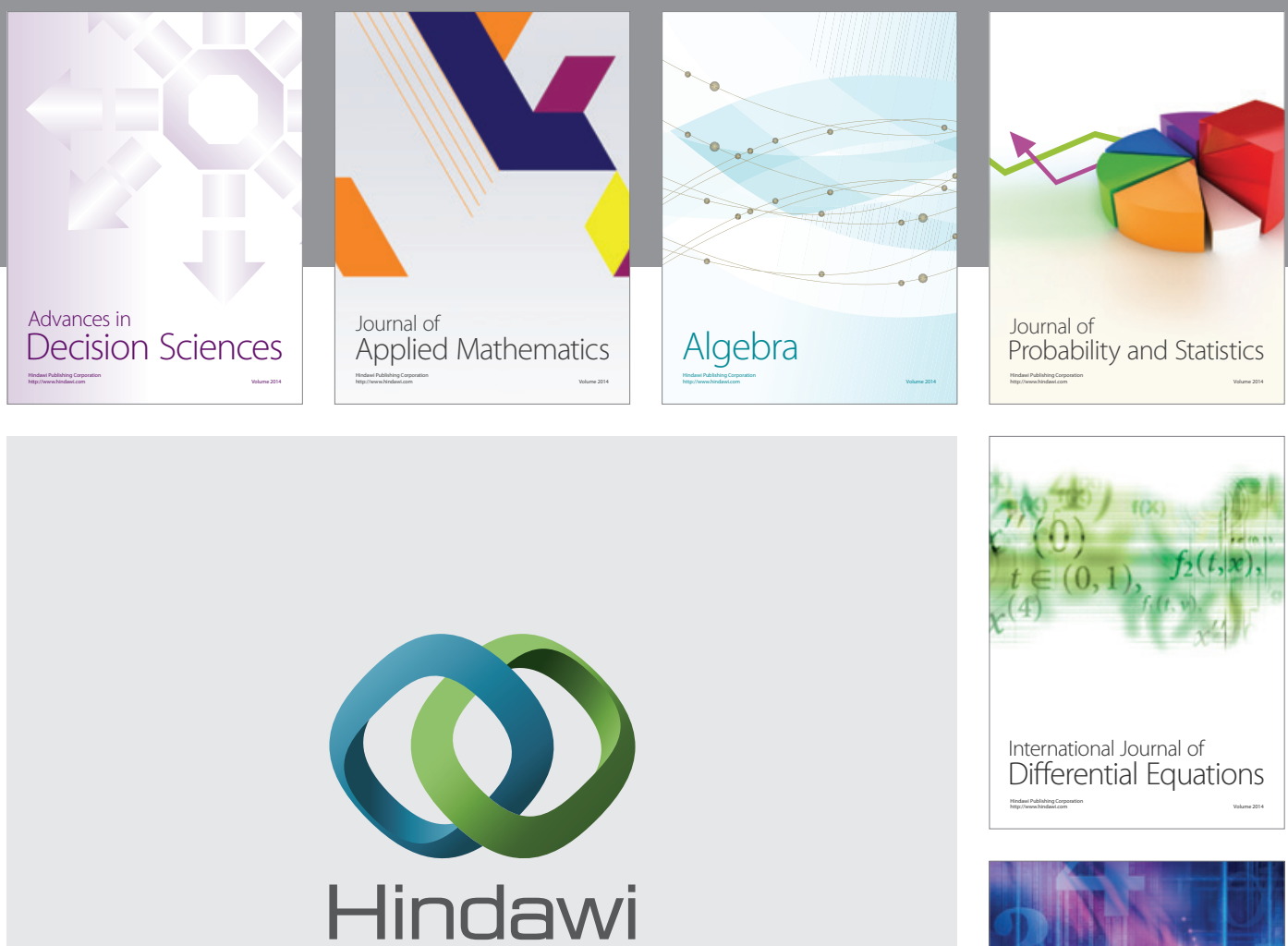

Submit your manuscripts at http://www.hindawi.com
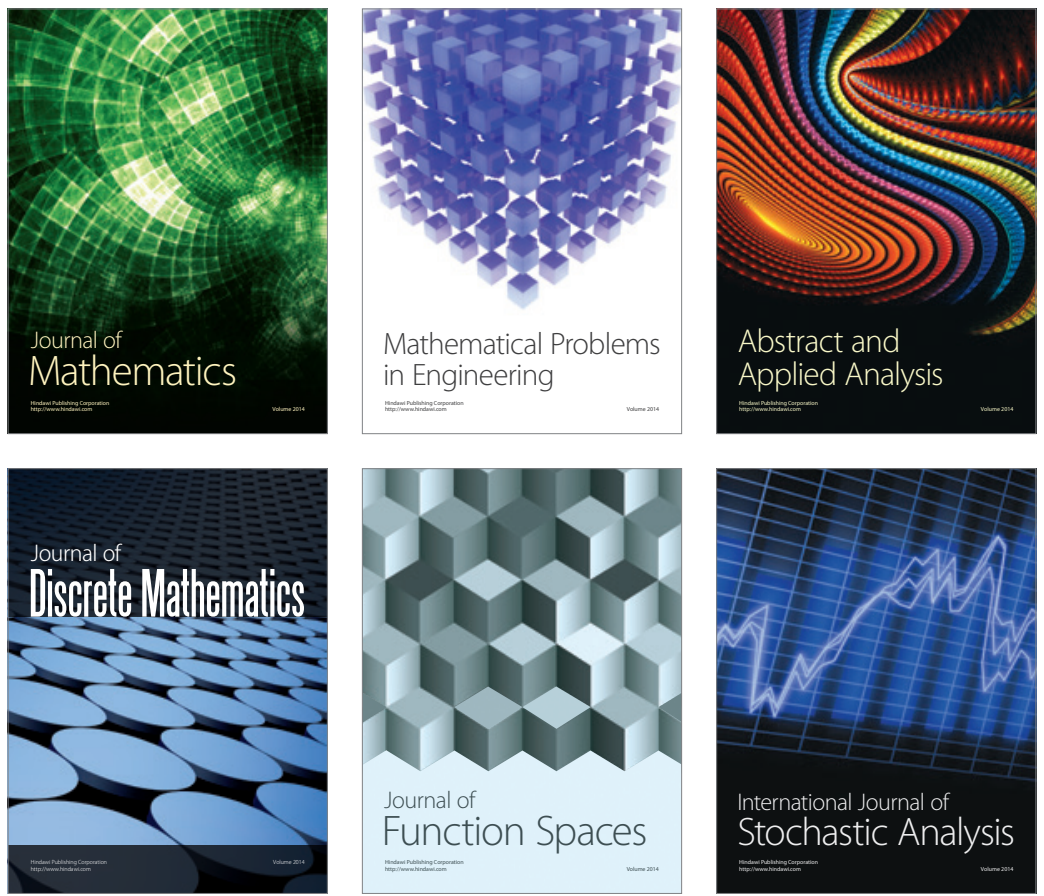

Journal of

Function Spaces

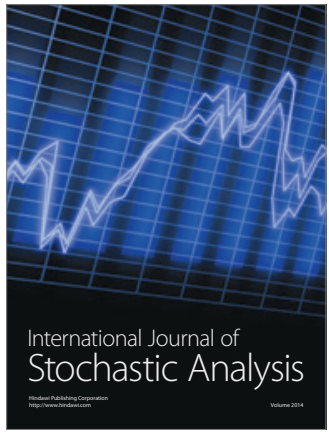

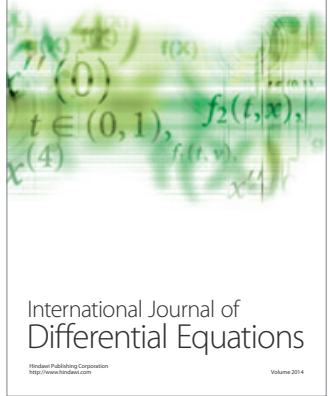
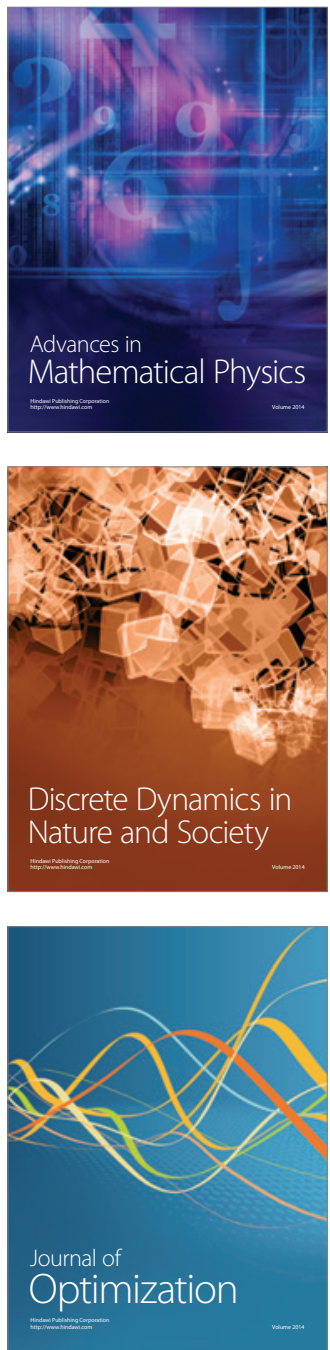